\title{
Desarrollo de la producción escrita en inglés al término de la Educación Secundaria Obligatoria española
}

\author{
Maria Dolores Corpas y Daniel Madrid \\ Facultad de CC de la Educación, Universidad de Granada
}

Recibido: 20-1-07 / Aceptado: 12-4-07

ISSN: $1697-7467$

\begin{abstract}
RESUMEN: Este artículo presenta una investigación llevada a cabo con estudiantes de cuarto de Educación Secundaria Obligatoria (ESO) a fin de conocer y reflexionar sobre el desarrollo de la producción escrita en el área de lengua extranjera, inglés, al término de esta etapa educativa en relación a tres objetivos específicos: conocer el desarrollo global de la producción escrita en lengua inglesa al terminar la ESO, conocer el desarrollo de elementos específicos de la producción escrita en lengua inglesa y determinar si existen diferencias según el género del alumnado en la producción escrita del alumnado. Finalmente, describimos el perfil del alumnado medio en producción escrita conforme al Marco Común Europeo.

Los resultados globales nos permiten concluir que solamente un $10 \%$ del alumnado consigue el objetivo que marca el MEC/Comunidad Autónoma y que las alumnas consiguen mejores resultados que sus compañeros, con una media de 2' 47 frente a 2'08 en una escala de 1 a 5 puntos. Esta diferencia es estadísticamente significativa según el estadístico de contraste no paramétrico U de Mann-Whitney.
\end{abstract}

Palabras Clave: producción escrita, evaluación, Educación Secundaria Obligatoria, género.

\begin{abstract}
The present article is a research study on English language written production in the fourth year of the Spanish Compulsory Secondary Education (ESO). It aims to analyse the writing development in this subject, at the end of this educational stage in relation to three specific objectives: knowing the global competency level of the ESO students in writing, to report about the development of specific elements in the ESO students' written production and studying the differences in their written production in relation to the students' gender. Finally, we describe the average student's characteristics in written production according to the Common European Framework.

The global results allow us to conclude that only $10 \%$ of the students achieve the objective established by the Local Authorities and that female students obtain better results than males students (a mean score of 2,47 for girls and 2,08 for boys in a scale from 1 to 5 points). These differences are statistically significant according to the $\mathrm{U}$ de Mann-Whitney's non-parametric statistical procedure.
\end{abstract}

Key words: written production, assessment, Compulsory Secondary Education, gender. 


\section{INTRODUCCIÓN}

Por nuestra propia experiencia como hablantes de una lengua, sabemos que la escritura es una actividad difícil y compleja. Los problemas surgen por razones psicológicas, lingüísticas y cognitivas. Desde el punto de vista psicológico, las razones se deben a que la escritura es una actividad en la que el interlocutor no está presente. Por este motivo no es posible la interacción ni la retroalimentación. Este hecho impone restricciones lingüísticas, puesto que exige compensar las carencias de ciertos elementos como la entonación. Cognitivamente, el individuo, según va creciendo va adquiriendo la comprensión oral y expresión oral, de forma natural, sin un esfuerzo consciente, a diferencia de la escritura, aprendida gracias a la instrucción.

$\mathrm{Si}$, como acabamos de ver, la escritura es compleja en la lengua materna, estas dificultades aumentan cuando se trata de una lengua extranjera, en la que la habilidad lingüística y comunicativa se encuentra en desarrollo, como señala Manchón et al (2005: 378):

(...) basically, L2 writers, as compared to their monolingual, counterparts, have less knowledge of the L2 (at some linguistic levels) and/ or less possibility of having automatic access to the L2 knowledge that they possess.

Debido a la complejidad que presenta la escritura en lengua extranjera y como profesores de esta asignatura, nos hemos planteado estudiar qué desarrollo de la producción escrita logra nuestro alumnado al término de la Educación Secundaria Obligatoria y si existe diferencia entre los chicos y las chicas. Tal y como marcan los objetivos de la Educación Secundaria Obligatoria para Andalucía, decreto 148/2002 de 14 de mayo, el alumnado debe desarrollar una serie de capacidades y destrezas. En lo que se refiere a la enseñanza de lenguas extranjeras y al desarrollo de la expresión escrita, el apartado j del mismo decreto afirma que el alumnado debe "comprender y producir mensajes orales y escritos contextualizados, en la lengua o lenguas extranjeras objeto de estudio" (BOJA de 27 de junio de 2002: 11470). En este trabajo informaremos sobre el grado de consecución de este objetivo, daremos a conocer algunas producciones del alumnado que ilustran el nivel de competencia escrita que consiguen y las diferencias encontradas en función de la variable sexo.

\section{LA PRODUCCIÓN ESCRITA}

Como hemos indicado, la producción escrita es una actividad compleja y muy diferente a la producción oral. Cuando nos expresamos oralmente, lo solemos hacer en un contexto situacional y, al menos, parte del lenguaje utilizado se refiere a esa situación. Nos estamos refiriendo al contexto físico, a características paralingüísticas como los gestos y la entonación, a rasgos prosódicos (acentuación, entonación, ritmo y pausas) y, sobre todo, a la posibilidad de rectificar o de aclarar, según las necesidades del interlocutor. En resumen, la interacción y la retroalimentación son continuas. Esta retroalimentación puede ser verbal o no verbal. La comunicación verbal puede estar constituida por un simple gruñido o llegar a ser un discurso extenso. No menos importante es la comunicación no verbal o paralingüística. 
Una simple expresión facial puede, por ejemplo, mostrar desacuerdo con lo que dice nuestro interlocutor.

Cuando escribimos, todo esto es diferente. El interlocutor no está presente, por lo que debemos suplir la carencia del contexto con otras herramientas, como advierte Byrne (1979:2):

Writing, by contrast, is the whole of the relevant activity and we create the context as we write. As we have noted, the person we are addressing is not present, so there can be no interaction between writer and reader. Although we may try to anticipate reactions and build them into the text, we have to sustain the whole process of communication on our own and to try to stay in contact with our reader through words alone.

Como Manchón et al.(2005: 383) indica, la ausencia de un soporte físico y contextualizado propicia la aparición de características propias de los textos escritos. A continuación, citamos las más importantes desde el punto de vista de estos autores:

- La permanencia. A diferencia del lenguaje oral, el escrito permite consolidarse en el tiempo. Esto implica que el texto puede ser releído, analizado y discutido. El destinatario decide el tiempo de su existencia. Además tiene la posibilidad de compartirlo con otros. En la enseñanza de lenguas, los textos escritos nos ayudan a ver la progresión del alumno a lo largo del tiempo.

- El tiempo dedicado a producir el texto. Los textos escritos exigen un mayor tiempo de creación. Se pueden releer, modificar o ampliar, antes de que el lector tenga acceso a ellos.

- La distancia. El lector de los textos escritos no suele compartir el contexto de la creación del texto. Es por eso que, el escritor debe anticiparse a las reacciones provocadas por su texto. Brown (2001:342) llama "empatía cognitiva" al hecho de leer el texto propio con los ojos del lector.

- La ortografía. A veces se plantean problemas al convertir el lenguaje oral en lenguaje escrito, puesto que no todos los fonemas se corresponden con una determinada grafía. La dificultad es directamente proporcional a la posibilidad de representación gráfica de los fonemas.

- Complejidad. La escritura es un proceso complejo porque requiere que el individuo sea lo suficientemente habilidoso para eliminar redundancias, unir ideas de manera lógica y con las conjunciones adecuadas, con una determinada gama de términos, entre otros elementos.

- Vocabulario. El vocabulario de los textos escritos es mayor que en los textos orales. Esto es así porque la escritura demanda una mayor densidad de nuevos vocablos para evitar problemas de redundancia, estilo, etc.

- Convencionalismos. El escritor debe seguir distintas convenciones específicas de cada cultura puesto que cada tipo de texto (por ejemplo, una instancia, una carta expresando nuestras quejas o una redacción escolar) exige un convencionalismo específico.

- Explicitud. Con la intención de evitar ambigüedades, el texto escrito debe ser explícito, supliendo las carencias contextuales presentes en el lenguaje oral.

- Instrucción. Tanto la escritura como la lectura son destrezas que deben ser aprendidas 
mediante la instrucción. No sucede lo mismo con el habla y con la audición, que se van desarrollando paralelamente al crecimiento del individuo.

La escritura, como destreza productiva, requiere de ciertos recursos con el objeto de extraer del potencial disponible aquél que se adapte a la naturaleza de la tarea. Así el Marco Común Europeo de las Lenguas (2001: 63) declara las siguientes etapas en el desarrollo de la producción escrita.

1) Planificación. Ésta incluye una preparación consciente, denominada ensayo, pensando en los efectos de los diferentes estilos, estructuras del discurso y formulaciones, teniendo en cuenta a la audiencia, además de sopesar la posibilidad de buscar determinadas herramientas, pedir ayuda, es decir, localizar distintos recursos. Si el apoyo necesario no ha sido localizado, el individuo puede modificar sus primeras intenciones, inclinándose por un texto más modesto y sencillo. Justamente lo contrario puede suceder si ha encontrado el material necesario. A esto se le llama ajustar la tarea. Lo mismo ocurre con el ajuste del mensaje. Dependiendo de los elementos lingüísticos disponibles el autor puede aumentar o disminuir su producción.

2) Ejecución. Si una vez analizados los recursos, el individuo decide no emplearlos, éste estaría utilizando una estrategia de evitación. De lo contrario, la estrategia sería positiva, o sea, de logro. Quizás necesite utilizar alguna técnica de ejecución como la compensación (utilizar un lenguaje en el que se sienta seguro), construir sobre sus conocimientos previos o realizar diversos intentos.

3) Evaluación. Después de haber producido el texto escrito, el individuo puede conscientemente evaluar sus errores lingüísticos y comunicativos.

4) Reparación. El último paso es corregir los errores detectados y producir una nueva versión.

Uno de los aspectos claves de la composición escrita es que sirve de refuerzo a las destrezas orales, refuerza las estructuras gramaticales y desarrolla la comunicación escrita (Raimes, 1983). También es fundamental la relación entre escritura y pensamiento en la LE y su incidencia en la competencia discursiva a la hora de conectar las oraciones de acuerdo con su relación funcional, temática y gramatical (White, 1983).

En los procesos de comunicación escrita hemos de destacar la escritura como "propósito"o respuesta a una demanda social y como actividad dirigida a un destinatario o lector de nuestro mensaje. En este sentido, hemos de tener en cuenta las fases que establece Sampson (1981) en la escritura con enfoque funcional:

- se trata de un producto final,

- se dirige a una audiencia específica,

- se realiza con un propósito,

- y se centra en unos aspectos lingüísticos.

A estos cuatro rasgos, le añadimos el vacío de información que se produce entre la información que tiene el que escribe y el que lee y la dependencia de la tarea, es decir, la relación y dependencia entre la respuesta del que lee y el texto leído. 
En las actividades de expresión escrita, debemos de ser conscientes de la importancia que tiene para el alumno que escribe el dirigirse a una audiencia real (Hedge, 1988; Flower, 1979), por ejemplo a través del chatting de Internet, del correo electrónico o del mismo profesor. Otro elemento clave consiste en activar los elementos lingüísticos y sociolingüísticos necesarios para que el mensaje sea correcto y adecuado (appropriate) a la situación.

Entre las funciones más comunes que se suelen expresar en los procesos de comunicación escrita, siguiendo a Sampson (1981) y Pincas (1983), podrían destacarse los siguientes (Bueno, 1996):

- escribir sobre uno mismo,

- dar instrucciones,

- narrar hechos en presente o en pasado,

- describir, predecir y planificar,

- explicar procesos,

- expresar secuencias de hechos,

- contrastar y expresar similitudes,

- dar argumentos a favor y en contra, etc.

Entre los productos finales que resalta Sampson (1981) encontramos: notas, cartas, postales, felicitaciones, historias y narraciones, discursos, instrucciones, anuncios, canciones y poemas, recetas, entrevistas, anécdotas, etc.

En la actualidad, ya no se considera la producción escrita como un producto final acabado (Hedge, 2000), sino que se pone el énfasis en el proceso (Bueno, 1996; Grabe y Kaplan, 1996; Manchón 1999 y 2001; Camps et al., 2000), en las fases que se suceden mientras se desarrolla la actividad escritora (pre-writing y editing). Es decir, se centra la atención en cómo escriben los alumnos, cómo planifican, organizan, revisan y vuelven a lo escrito para corregirlo hasta llegar a la versión final (Lewitt, 1990 y Hedge, 1988). En este sentido, conviene comprender que los procesos de escritura nos son lineales, sino recursivos. A continuación, pasamos a describir brevemente esas etapas, que guardan cierto paralelismo con las que hemos mencionado anteriormente dentro del Marco de Referencia Europeo (2001:63) (véase también Bueno, 1996 y 2005; Manchón et al. 2005):

- Planificación y organización de lo que se va a escribir. En este apartado son fundamentales algunas recomendaciones de Raimes (1983), Márquez (1981) y Crewe (1990).

- Actividades previas a la composición: expresar mediante frases y oraciones las ideas que se desean expresar, desarrollo del esquema organizativo del contenido (Ike, 1990).

- Borrador: Primera versión del texto para que la revise el profesor o el compañero y sugieran posibles cambios; lectura, revisión y análisis de lo escrito para ver cómo se podría mejorar (Chenoweth, 1987).

- Edición: toque final y elaboración del texto definitivo (Lewitt, 1990)

\subsection{Recursos y técnicas para el desarrollo de la expresión escrita en LE}

Respecto a los recursos que ayudan a desarrollar la producción escrita en la Educación Secundaria, recomendamos la integración de la lectura y escritura (Aboderin, 1986; Bueno, 
1996) mediante el uso de textos que se leen primero y se emplean después para realizar la composición escrita. El input y output oral del alumnado es también una buena fuente para la escritura y se contribuye así a la consolidación del lenguaje estudiado oralmente. El uso de material visual es un recurso clásico que ayuda mucho a componer textos de acuerdo con los motivos que se ilustran.

Entre las técnicas que recomendamos, podríamos citar la composición cooperativa por parejas y en grupo (Ike, 1990). Otras técnicas ayudan a planificar y a pensar en la posible organización de los textos: brainstorming, debates y discusiones, las entrevistas, etc. Para que el alumnado aprenda a organizar los textos, recomendamos la elaboración de esquemas, la identificación del tema de los párrafos que puede servir de guía, de los elementos generales y específicos, de las ideas principales y secundarias, etc. Como técnicas procesuales que pueden mejorar los borradores escritos, no debemos olvidar el trabajo cooperativo con otros compañeros, pedir ayuda al profesor, transferir elementos de unos textos a otros, evitar expresar determinadas ideas complejas o simplificarlas (avoidance), etc.

Pero el proceso de la producción escrita no termina hasta que el texto llega a su destinatario o a otro lector y se produce una respuesta. Esta respuesta, cuando el destinatario es el profesor, suele ir acompañada de una corrección y de su correspondiente calificación. En la lectura que verifica el destinatario, lo más relevante es el significado (Dixon, 1986); lo que se transmite y los defectos formales, aunque también son importantes, pasan a un segundo plano. En esta fase final, Raimes (1983) resalta la importancia de la revisión guiada por parte del profesor y de la autoedición del propio alumno aplicando los consejos que haya recibido y Charles (1990) recomienda la auto-monitorización por parte de los alumnos: los alumnos escriben y monitorizan lo que hacen y el profesor responde a esos comentarios. En esta fase es también importante la revisión de los propios compañeros de clase y los comentarios que formulen para mejorar las composiciones, que puede usarse a modo de feedback por el profesor (véase Ferris, 2004 y Golstein, 2004).

En relación a las técnicas de expresión escrita que se practican en las aulas, hemos de destacar los ejercicios de composición guiada, controlada y libre y de otras muchas técnicas que contribuyen a su desarrollo, como se ilustra en Madrid y McLaren (1995). De especial interés es la actividad metalingüística durante el proceso de la escritura, es decir, la conexión entre la actividad escritora y su aprendizaje, siguiendo las premisas establecidas por Camps et al. (2000). Es decir, el hecho de que:

- Tanto los procesos de producción como los de comprensión lingüística implican cierta habilidad metalingüística para analizar y controlar el lenguaje.

- Las representaciones lingüísticas se construyen en el marco de la interacción social, ya que es a través de estas interacciones cómo se activa el aprendizaje lingüístico y la construcción de su conocimiento.

- La escuela y el entorno lingüístico se convierten en el escenario donde se desarrolla el lenguaje y donde tiene lugar la adquisición y el dominio de los usos lingüísticos y de los conceptos que se asocian con el conocimiento del código elaborado (Berstein, 1971) o del CALP (Cognitive/Academic Language Proficiency, Cummins, 1979).

- El trabajo colaborativo es una herramienta eficaz para activar la actividad metalingüística.

- La actividad metalingüística explícita que se origina a través de la interacción social puede ir acompañada del uso de un metalenguaje específico. 
- La actividad metalingüística incluye tanto el uso de términos específicos como el uso de lenguaje cotidiano.

\section{Algunos estudios sobre la producción escrita del alumnado de INGLÉS COMO L.E.}

El Instituto Nacional de Calidad y Evaluación (INCE) de España junto con la Dirección de Evaluación y de la Prospectiva (DEP) de Francia y la Agencia Nacional de Educación (Skolverket) de Suecia han realizado una evaluación comparativa e internacional sobre los conocimientos y competencias adquiridas en inglés por el alumnado de edades comprendidas entre los 15 y 16 años, cuyos datos fueron expuestos en 1987 en París. De los datos obtenidos en producción escrita, se deduce que el alumnado francés evaluado alcanza los objetivos propuestos en un $41 \%$. El español se queda dos puntos por debajo en un 39\%. El alumnado sueco es el que mejor resultado logra, con una media del $60 \%$.

En 2002, el estudio sobre la Evaluación del Rendimiento de los Alumnos en Inglés en Ocho Países Europeos Francia, España, Suecia, Dinamarca, Noruega, Alemania, Finlandia y Países Bajos es una ampliación del anterior proyecto. La producción escrita logra los resultados más bajos de todos los evaluados. De hecho, los resultados más altos rondan el $50 \%$ de aciertos de media. Los alumnos franceses son los que presentan peores resultados $\left(14{ }^{\prime} 55 \%\right)$, seguidos por los españoles (23’41\%).

El INCE publicó un informe titulado La Evaluación de la Enseñanza y el Aprendizaje de la Lengua Inglesa en Educación Secundaria Obligatoria 2001. En síntesis, los objetivos generales fueron conocer y valorar las capacidades básicas logradas por el alumnado de la Educación Secundaria Obligatoria (ESO), al término del primer y segundo ciclo, con respecto al nivel de comunicación, conocimientos de la lengua inglesa adquiridos y conocer los factores del proceso enseñanza-aprendizaje que influyen en los resultados del alumnado. En la prueba de producción escrita, se valoró la corrección y la adecuación semántica. La primera incluye la ortografía y un correcto uso gramatical. La segunda evalúa la capacidad del alumnado para utilizar un vocabulario adecuado al texto. Los porcentajes medios obtenidos nos indican que el 39\% consiguieron este objetivo. Un punto porcentual menos obtiene la corrección. Muy por encima se encuentra la puntuación de la adecuación que se situó en torno al $60 \%$.

En el estudio que presentamos a continuación, hemos empleado mayor variedad de técnicas para evaluar la producción escrita de los estudiantes y hemos incluido un "Project" para que el alumnado demuestre su capacidad para buscar información, sistematizarla y reflejarla en su composición escrita.

\section{Diseño Del estudio}

En este estudio nos hemos propuesto los siguientes objetivos:

\subsection{Objetivos}

A. Conocer el desarrollo global de la producción escrita en lengua inglesa, al terminar la Educación Secundaria Obligatoria. 
B. Conocer el desarrollo de elementos específicos de la producción escrita en lengua inglesa al terminar la Educación Secundaria Obligatoria (contenido, cohesión y coherencia, registro, corrección lingüística y de errores, reconocimiento y producción de palabras y ortografía).

C. Determinar si existen diferencias según el género del alumnado en la producción escrita en lengua inglesa.

\subsection{Población}

La población de este estudio está compuesta por el alumnado que cursa la materia de inglés en cuarto de ESO (16 años) en un Instituto andaluz de Enseñanza Secundaria. La muestra real ${ }^{1}$, compuesta por noventa y cuatro alumnos, cuarenta y ocho chicas y cuarenta y seis chicos, se ve mermada con respecto a la muestra invitada debido a que, algunos alumnos abandonaron sus estudios antes de que la prueba les fuera pasada y a que algunos de ellos no la concluyeron por distintos motivos. Los datos de la población se muestran en la tabla siguiente:

\begin{tabular}{lccc}
\hline & Chicas & Chicos & Total \\
\hline Muestra Invitada & 51 & 47 & 98 \\
Muestra Participante & 50 & 47 & 97 \\
\hline Muestra Real & 48 & 46 & 94 \\
\hline
\end{tabular}

Hemos de destacar que todos los estudiantes iniciaron el estudio del inglés desde el comienzo de la escolaridad, a los 6 años. El porcentaje de fracaso escolar de la muestra en relación al rendimiento obtenido en la asignatura Inglés se sitúa casi en el 30\%, el 60\% se sitúa en una franja de rendimiento intermedio con una calificación de aprobado o bien y el $10 \%$ restante consigue buenos resultados (notable o sobresaliente)

\subsection{Instrumentos para la recogida de datos}

El objetivo de las pruebas es que el alumno produzca, de forma eficaz, distintos tipos de textos con diversas finalidades, en situaciones de comunicación pasadas, habituales o futuras utilizando diversos recursos lingüísticos. La prueba consta de diez tareas (ver anexo I), con un total de treinta y un ítems. La extensión recomendada de los textos fue de cien palabras en cada tarea. La duración del conjunto de las tareas fue de cincuenta y cinco minutos. Además, una submuestra del alumnado, diecisiete individuos elegidos al azar, realizó un proyecto (Project), consistente en desarrollar un tema utilizando diversos materiales

${ }^{1}$ La muestra invitada, constituida por los individuos de la población a quienes se les invita a participar, está formada por cincuenta y una alumnas y por cuarenta y siete alumnos. En la muestra participante, compuesta por los sujetos que aceptan formar parte del estudio, disminuye en una alumna con respecto a la muestra invitada. 
como Internet, libros de consulta, etc. La duración de esta tarea fue de cincuenta y cinco minutos. A continuación exponemos una descripción detallada de las tareas (ver anexo I).

\section{Primera Tarea}

Objetivo: Comunicarse por escrito de manera eficaz en situaciones habituales de comunicación a través de tareas específicas.

Formato: Se le propone al alumno una situación problemática ocurrida durante un viaje. Tipo de texto: Narración del problema que se le ha planteado.

Actividad: El alumno debe escribir un texto con una extensión máxima de cien palabras, en el que se exponga un problema ocurrido en un viaje.

Número de preguntas: Una única cuestión.

\section{Segunda Tarea}

Objetivo: Comunicarse por escrito de manera eficaz en situaciones habituales o futuras de comunicación

Formato: Se presenta un texto para que el alumnado lo utilice como modelo en la realización de la tarea.

Tipo de texto: Descripción de la ropa favorita del alumno.

Actividad: La actividad consta de dos partes. En primer lugar, el alumno debe leer un texto que se le presenta como modelo para posteriormente utilizarlo como modelo en la descripción de su propia ropa.

Número de preguntas: Una única cuestión.

\section{Tercera Tarea}

Objetivo: Producir mensajes escritos en situaciones habituales de comunicación utilizando distintos recursos.

Formato: Al alumno se le da una serie de instrucciones para que produzca un texto escrito.

Tipo de texto: Descripción de un lugar.

Actividad: El alumno debe producir un texto escrito sobre el lugar en el que vive, según las instrucciones.

Número de preguntas: Una pregunta.

\section{Cuarta Tarea}

Objetivo: Reflexionar sobre el funcionamiento lingüístico.

Formato: Oraciones en las que se ha cometido distintos errores.

Tipo de texto: Cinco oraciones.

Actividad: El alumno debe corregir los errores que aparecen subrayados en distintas oraciones.

Número de preguntas: Cinco preguntas.

\section{Quinta Tarea}

Objetivo: Reflexionar sobre el funcionamiento semántico de la lengua extranjera

Formato: En esta tarea existen dos formatos diferenciados. Por un lado, una pregunta de elección múltiple. Por otro, debe escribir el sinónimo palabras dadas. 
Tipo de texto: Palabras pertenecientes a un campo semántico.

Actividad: El alumno debe señalar de una serie de cuatro palabras la que no pertenezca al campo semántico. Además, debe proporcionar un sinónimo de otras palabras.

Número de preguntas: Ocho palabras.

\section{Sexta Tarea}

Objetivo: Reflexionar sobre el funcionamiento lingüístico y comunicativo de la lengua extranjera.

Formato: Texto con huecos en una misma palabra y texto con errores ortográficos.

Tipo de texto: Oraciones con omisión de ciertas vocales.

Actividad: El alumno debe completar una serie de oraciones en las que se ha omitido algunas vocales. Se ofrece las posibles combinaciones entre vocales. Además, debe corregir la ortografía de las palabras que forman varias oraciones.

Número de preguntas: 23 palabras con dificultad ortográfica en 6 oraciones.

\section{Séptima Tarea}

Objetivo: Reflexionar sobre los propios procesos de aprendizaje.

Formato: Elegir de una serie de ideas aquellas que el estudiante utilice en su proceso de aprendizaje.

Tipo de texto: Pedagógico.

Actividad: Responder a una serie de preguntas teniendo en cuenta una serie de ideas previas.

Número de preguntas: Dos preguntas.

\section{Octava Tarea}

Objetivo: Leer de forma comprensiva un texto con la intención de apreciar los significados sociales y culturales que transmiten la lengua inglesa y escribir un texto relacionado con éste.

Formato: Un texto y una cuestión relativa al texto.

Tipo de texto: Descripción de una festividad.

Actividad: El alumnado debe leer el texto y resolver una cuestión.

Número de preguntas: Una única pregunta.

\section{Novena Tarea}

Objetivo: Valorar por escrito la importancia de las lenguas extranjeras.

Formato: Cuestiones sobre la importancia de las lenguas extranjeras.

Tipo de texto: Preguntas para que el alumno dé su opinión sobre la importancia de las lenguas.

Actividad: El alumno debe responder a una serie de cuestiones.

Número de preguntas: Tres.

\section{Décima Tarea}

Objetivo: Valorar por escrito la importancia del conocimiento de otras lenguas.

Formato: Al alumno se le plantea un problema que debe resolver.

Tipo de texto: Texto argumentativo. 
Actividad: Al alumno se le pide que imagine que está organizando un congreso médico en Bruselas y, como responsable del mismo, debe elegir dos lenguas oficiales, justificando y argumentando su respuesta.

Número de preguntas: Una.

\section{Undécima Tarea ( Project $\left.^{2}\right)$}

Objetivo: Transferir a la lengua extranjera las estrategias de comunicación adquiridas en la lengua materna y utilizar estrategias de aprendizaje y recursos didácticos para narrar acontecimientos.

Formato: Presentada una cuestión, el alumno debe resolverla.

Tipo de texto: Texto descriptivo y narrativo.

Actividad: El alumno debe describir o narrar acontecimientos relevantes para el mundo o para su país ocurridos en la década en la que nació. Para documentarse, puede utilizar recursos didácticos como enciclopedias, libros de historia, Internet etc.

Número de preguntas: Una única cuestión.

\subsection{Criterios de Corrección}

A la hora de analizar y evaluar las actividades de la prueba escrita del alumnado, hemos tenido en cuenta varios criterios de tipo lingüístico, sociolingüístico y pragmático que, en su conjunto y de forma integrada, constituyen las dimensiones fundamentales de la producción textual y de la habilidad escritora (véase Raimes, 1983 y Manchón et al. 2005). Hemos utilizado una escala de uno a cinco puntos, donde uno equivale a muy deficiente, dos a insuficiente, tres a suficiente, cuatro a notable y cinco a sobresaliente, en cada uno de los siguientes criterios de corrección:

- Contenido de la tarea:

El alumno debe producir textos con información relevante, necesaria y significativa conforme al enunciado de la tarea. Se ha aplicado en la tarea 1, 2, 3, 7, 8, 9, 10 y 11.

- Organización y cohesión

Los textos deben regirse por un orden en la disposición de las ideas y una unión entre ellas mediante diferentes recursos como conjunciones, deixis, sinónimos, etc. Se ha aplicado en las tareas siguientes: 1, 2, 3, 8, 10 y 11.

- Registro

El alumnado debe expresarse con un registro adecuado, adoptado según una configuración situacional y particular del tema, del modo y de los participantes. Se ha aplicado en las siguientes tareas: $1,2,3,8,9,10$ y 11 .

- Corrección lingüística

El lenguaje debe ser apropiado para cada tarea (narración, descripción, argumentación, etc.) y la expresión correcta, libre de errores semánticos, ortográficos, sintácticos o pragmáticos. Se ha aplicado en la tarea 1, 2, 3, 8, 9, 10 y 11.

2 Esta actividad ha sido desarrollada sólo por una parte del alumnado, concretamente por diecisiete alumnos y alumnas elegidos al azar, debido a problemas de espacio y tiempo. Dispusimos de la biblioteca y de todas sus instalaciones. Para la realización de la prueba, tuvieron cincuenta y cinco minutos. 
- Corrección de errores

La corrección de errores implica enmendar lo errado, reflexionando sobre las formas y uso de la lengua. La corrección de errores es fundamental cuando el alumnado revisa sus producciones y las edita para mejorar su redacción. Se ha aplicado en la tarea 4.

- Reconocer la palabra adecuada según el contexto

Con esto, pretendemos que el alumno demuestre que es capaz de elegir una palabra de entre varias de una serie, sinónima a la dada. Esta habilidad influye a la hora de elegir el vocabulario más apropiado para el tipo de texto que desea escribir y es fundamental en el examen de inglés de la Selectividad para el ingreso en la Universidad española. Se ha aplicado en la tarea 5.

- Escribir la palabra adecuada según el significado

El individuo debe ser capaz de producir palabras adecuadas según los significados que desea expresar. Se ha aplicado en la tarea 5.

- Ortografía

El alumnado debe escribir conforme al conjunto de normas que regulan la escritura de la lengua inglesa. Se ha aplicado en la tarea 6.

\subsection{Tratamiento estadístico}

Para el estudio estadístico, hemos utilizado la versión 14.0 del programa SPSS (Statitical Package for the Social Science) para Windows.

\subsection{Validez y Fiabilidad}

La validez ha sido avalada por un juicio de expertos, usando el profesorado de inglés del Seminario del Instituto. Para determinar el grado de fiabilidad, hemos utilizado la prueba Alfa de Cronbach ${ }^{3}$.

\section{- Expresión escrita (writing) (todas las tareas menos el "Project")}

Resumen del procesamiento de los casos

\begin{tabular}{l|c|c|}
\hline & $\mathrm{N}$ & $\%$ \\
\hline Casos válidos & 94 & 100 \\
\hline Casos excluidos* & 0 & 0 \\
\hline Toral & 94 & 100 \\
\hline
\end{tabular}
* eliminación por lista basada en todas las variables del
procedimiento

3 Coeficiente de fiabilidad estadística Alfa de Cronbach: es una prueba estadística basada en análisis de correlaciones que indica el grado de fiabilidad estadística de las respuestas a una escala de un número determinado de ítems. Sus valores límite están entre 0 y 1. 
Estadísticos de fiabilidad

\begin{tabular}{|l|l|}
\hline $\begin{array}{l}\text { Alfa de } \\
\text { Cronbach }\end{array}$ & $\begin{array}{l}\mathrm{N}^{\mathrm{o}} \mathrm{de} \\
\text { elementos }\end{array}$ \\
\hline .95 & 28 \\
\hline
\end{tabular}

Un valor $\pm=0,950$ nos indica una excelente fiabilidad.

\section{- Expresión escrita (writing), Project}

Resumen del procesamiento de los casos

\begin{tabular}{|l|l|l|}
\hline & $\mathrm{N}$ & $\%$ \\
\hline Casos válidos & 17 & 18,1 \\
\hline Casos excluidos* & 77 & 81,9 \\
\hline Total & 94 & 100 \\
\hline
\end{tabular}

* eliminación por lista basada en todas las variables del procedimiento

\begin{tabular}{|c|c|}
\hline $\begin{array}{c}\text { Alfa de } \\
\text { Cronbach }\end{array}$ & $\begin{array}{c}\mathrm{N}^{\circ} \text { de } \\
\text { elementos }\end{array}$ \\
\hline .93 & 4 \\
\hline
\end{tabular}

Un valor $\pm=0,93$ nos indica que una la fiabilidad es excelente.

\section{Resultados}

Los resultados de la tabla siguiente nos muestran el porcentaje de éxito del alumnado en la realización de las tareas de writing y en qué grado han conseguido los sub-objetivos que se propone evaluar cada ejercicio: 


\begin{tabular}{|c|c|c|c|c|c|c|}
\hline \multirow[t]{2}{*}{ Tareas y sub-objetivos } & \multicolumn{3}{|c|}{$\begin{array}{l}\text { \% que han } \\
\text { conseguido } \\
\text { el objetivo }\end{array}$} & \multicolumn{3}{|c|}{$\begin{array}{l}\text { \% que NO han } \\
\text { conseguido } \\
\text { el objetivo }\end{array}$} \\
\hline & chicos & chicas & total & chicos & chicas & total \\
\hline $\begin{array}{l}\text { Tarea 1: Comunicarse por escrito de manera eficaz } \\
\text { en situaciones habituales de comunicación a través de } \\
\text { tareas específicas. }\end{array}$ & 23,9 & 31,3 & 27,7 & 76,1 & 68,8 & 72,3 \\
\hline $\begin{array}{l}\text { Tarea 2: Comunicarse por escrito de manera eficaz } \\
\text { en situaciones habituales o futuras de comunicación }\end{array}$ & 39,1 & 60,4 & 50 & 60,9 & 39,6 & 50 \\
\hline $\begin{array}{l}\text { Tarea 3: Producir mensajes escritos en situaciones } \\
\text { habituales de comunicación utilizando distintos } \\
\text { recursos. }\end{array}$ & 15,2 & 39,6 & 27,7 & 84,8 & 60,4 & 72,3 \\
\hline $\begin{array}{l}\text { Tarea 4: Reflexionar sobre el funcionamiento } \\
\text { lingüístico. }\end{array}$ & 10,9 & 22,9 & 17 & 89,1 & 77,1 & 83 \\
\hline $\begin{array}{l}\text { Tarea 5: Reflexionar sobre el funcionamiento } \\
\text { semántico de la lengua extranjera }\end{array}$ & 13 & 20,8 & 17 & 87 & 79,2 & 83 \\
\hline $\begin{array}{l}\text { Tarea 6: Reflexionar sobre el funcionamiento } \\
\text { lingüístico y comunicativo de la lengua extranjera. }\end{array}$ & 78,3 & 81,3 & 79,8 & 21,7 & 18,8 & 20,2 \\
\hline $\begin{array}{l}\text { Tarea 7: Reflexionar sobre los propios procesos de } \\
\text { aprendizaje. }\end{array}$ & 63 & 72,9 & 68,1 & 37 & 27,1 & 31,9 \\
\hline $\begin{array}{l}\text { Tarea 8: Leer de forma comprensiva un texto con la } \\
\text { intención de apreciar los significados sociales y } \\
\text { culturales que transmiten la lengua inglesa y escribir } \\
\text { un texto relacionado con éste. }\end{array}$ & 6,5 & 31,3 & 19,1 & 93,5 & 68,8 & 80,9 \\
\hline $\begin{array}{l}\text { Tarea 9: Valorar por escrito la importancia de las } \\
\text { lenguas extranjeras. }\end{array}$ & 8,7 & 16,7 & $12, .8$ & 91,3 & 83,3 & 87,2 \\
\hline $\begin{array}{l}\text { Tarea 10: Valorar por escrito la importancia del } \\
\text { conocimiento de otras lenguas. }\end{array}$ & 4,3 & 20,8 & 12,8 & 95,7 & 79,2 & 87,2 \\
\hline $\begin{array}{l}\text { Tarea } 11 \text { (Project): Transferir a la lengua extranjera } \\
\text { las estrategias de comunicación adquiridas en la } \\
\text { lengua materna y utilizar estrategias de aprendizaje y } \\
\text { recursos didácticos para narrar acontecimientos. }\end{array}$ & 6,5 & 6,3 & 6,4 & 93,5 & 93,8 & 93,6 \\
\hline
\end{tabular}

\section{Nivel máximo}

Los niveles más altos de producción escrita que hemos obtenido al final de la Educación Secundaria Obligatoria se pueden apreciar en los ejemplos siguientes de textos realizados por el alumnado en el test:

Tarea 1: A brief composition about a problem on a journey...

When I went to the beach last summer my car had a problem. It was in the middle on the road. My father and my mother telephoned to the police. The police went to the road and they did own a favour. After three days, my car hasn't got a problem and my family and I went to my house.

Tarea 2: Writing about what the students wears after using a text as a model:

Eventually, I wear sport clothes.

When I'm go to school, I never wear skirt but I always wear jeans, tracksuit bottoms, t-shirts, trainers... 
When I'm go to the disco and go out with my friends, I wear skirt and boots. When I' $m$ relaxing at home, I wear confortable clothes (tracksuits bottoms, trainers or my pyjama). I love go our for a walk or to the shops because I love buy new clothes!

Tarea 3: A description of a village, town, city...

My town is Lucena (Córdoba).

There are a lots of curchies (for example San Mateo, Santo Domingo...) and a lots of places to pass a good time (for example el Coso, la Plaza Nueva, Multicines, Bolera, El Paseo de Rojas...)

My town has pollution because people have two or three cars/family.

Lucena has got a pavilion and a swimming-pool. In my opinion Lucena is a good town.

Tarea 8: Describing any celebration or cultural event using a reading text as a model:

In February people celebrate the Carnival. People wear fancy dresses and make jokes on the street.

People sing "Chirigotas" in the street.

It's fantastic!

\section{Nivel intermedio}

El nivel de competencia medio en inglés escrito que alcanza el alumnado con un rendimiento intermedio de aprobado se puede apreciar en los textos siguientes:

Tarea 1: A brief composition about a problem on a journey...

I went with my parent to Nerja for reading "El balcón de Europa" the past year. My car has a problem in a street and my father didn" + know this twon. My father saw a person and he asked for a mechanic but this day has feria in Nerja.

We was a day for the street of Nerja between the day siguiente

Tarea 2: Writing about what the student wears following a reading text as a model:

When I'm going to school, I wearing a jeans, t-shirt and trainer. When I going out my friends at weekend, I wearing a drees, skirt, pants, trousers and shoes.

Tarea 3: A description of a village, town, city...

I live in Lucena a twon of Cordoba.

I think that Lucena is very small and I don't like because Lucena haven't got a lot of clothes shops and discoteques.

In my opinion Lucena would had a lot of clothes hops and discoteques.

Tarea 8: A celebration or cultural event:

San Juan is celebrate in June. The people go to the beach for do fire.

In San Juan at $120^{\prime}$ clock of the night the boys and girls, swimming in the sea.

Es evidente que el nivel de competencia escrita desarrollado por el alumnado, después de haber estudiado inglés, en contextos de aula, durante 10 años es bastante pobre y equivale quizás a lo que deberían haber sido capaces de hacer al final de la Educación Primaria de 
acuerdo con los objetivos que marcan los diseños curriculares para esa etapa, donde se indica que deberán ser capaces de producir textos escritos breves sobre temas familiares, respetando las reglas básicas del código escrito. Aunque se trata del estudio de un caso que incluye una muestra cercana al centenar de estudiantes, creemos que los resultados obtenidos en este Instituto son generalizables y pueden ilustrar los niveles de inglés escrito que se consiguen al final de la Educación Secundaria Obligatoria en los centros públicos españoles. No cabe duda de que la facilidad con la que se ha llevado a cabo la promoción automática del alumnado a lo largo de la Educación Primaria y Secundaria con la LOGSE, la metodología de enseñanza que predomina en los centros y los niveles de exigencia que se piden son muy cuestionables y necesitan una revisión urgente.

Seguidamente, ofrecemos los datos referidos a los distintos criterios de corrección empleados para juzgar el desarrollo de la escritura en los estudiantes. Como podemos observar, la ortografía, con 3'01, es el único aspecto que alcanza un resultado positivo. La destreza que consiste en escribir la palabra adecuada según el significado ha obtenido la puntuación más baja, con un 1'51.

Puntuación media de los criterios de corrección empleados en producción escrita (writing, escala de 1 a 5 puntos)

- Contenido: 2'66

- Organización y cohesión: 2’36

- Registro: 2'18

- Corrección lingüística: 2’07

- Corrección de errores: 1'54

- Reconocer la palabra adecuada según el significado: 2'22

- Escribir la palabra adecuada según el significado: 1'51

- Ortografía: 3,01

\section{Resultados globales y SEgún LA VARIABLE GÉNERo}

Considerando la variable género, podemos afirmar que las chicas logran mejores resultados globales que sus compañeros, un 2'47 frente a un 2'08. Esta diferencia es estadísticamente significativa según la prueba U de Mann-Whitney ${ }^{4}$ La media global de la producción escrita en lengua extranjera en relación a la variable género es de 2’28, como puede apreciarse en la tabla siguiente:

${ }^{4}$ U de Mann-Whitney: es un estadístico de contrate no paramétrico que se usa para estudiar una variable ordinal o las medias de una escala en función de dos muestras independientes (equivalentemente, dos categorías de respuesta de una misma variable nominal u ordinal). 


\begin{tabular}{|c|c|c|}
\hline Género & \multicolumn{2}{|c|}{ Producción Escrita } \\
\hline \multirow[t]{3}{*}{ Chico } & Media & 2'08 \\
\hline & $\mathrm{N}$ & 46 \\
\hline & Desv. típ &, 64 \\
\hline \multirow[t]{3}{*}{ Chica } & Media & 2'47 \\
\hline & $\mathrm{N}$ & 48 \\
\hline & Desv. típ & ,75 \\
\hline \multirow[t]{3}{*}{ Total } & Media & $2 ’ 28$ \\
\hline & $\mathrm{N}$ & 94 \\
\hline & Desv. típ & 72 \\
\hline
\end{tabular}

\section{Conclusiones}

Con respecto a los objetivos planteados, los resultados obtenidos nos permiten extraer las siguientes conclusiones:

A) Desarrollo global de la producción escrita en lengua inglesa, al terminar la Educación Secundaria Obligatoria.

Solamente el 10'6\% de los estudiantes consigue producir escritos contextualizados en la lengua objeto de estudio y el $89^{\prime} 4 \%$ restante no lo consigue. Los resultados globales indican que el alumnado no logra el objetivo que marcan los diseños curriculares en producción escrita en lengua inglesa al término de su Educación Secundaria Obligatoria, (la nota media se sitúa en 2'28 en una escala de 1 a 5 puntos). Después de 10 años de estudio del idioma inglés, las producciones escritas del alumnado son pobres de contenido e insuficientes en cuanto a su cohesión y coherencia.

B) Desarrollo de elementos específicos de la producción escrita en lengua inglesa, al terminar la Educación Secundaria Obligatoria.

El alumnado no logra superar ningún criterio de corrección adoptado excepto la habilidad ortográfica. Tanto el contenido, como el registro empleado, la cohesión y coherencia de los textos y su capacidad de editar y corregir errores es deficiente. La extensión media de los textos se sitúa en torno a las 50 palabras, justamente la mitad de la extensión recomendada.

C) Diferencias según sexo en la producción escrita en lengua inglesa.

Las alumnas consiguen mejores resultados que sus compañeros, 2'47 frente a 2'08. Esta diferencia es estadísticamente significativa según la prueba U de Mann-Whitney.

Atendiendo a los niveles del Marco Común Europeo, el alumno medio de esta investigación pertenece al nivel A2. Es capaz de escribir una serie de oraciones simples unidas con conjunciones sencillas como "and", "but" o " because" sobre su familia, entorno y su educación. Puede escribir textos sencillos en presente, pasado y futuro describiendo sus intereses, 
acontecimientos simples y sus expectativas de futuro. No obstante, le resulta muy difícil expresarse correctamente desde el punto de vista lingüístico. Sus errores gramaticales son frecuentes, lo que, a veces, impide la comprensión del texto. La mayoría de las veces es incapaz de escribir el vocablo adecuado según el contexto y suele preferir las estrategias de evitación a las de logro. Como puede apreciarse en los ejemplos anteriores, tienen dificultades serias para producir textos escritos organizados, coherentes y cohesionados.

\section{Bibliografía}

Aboderin, Y. (1986). "Integrating reading and writing". English Teaching Forum 24/1: 38-40.

Alderson, J. C, Clapham C. y Wall, D. (1998). Exámenes de idiomas, elaboración y evaluación.Traducción de Neus Figueras. Madrid: Cambridge University Press.

Allen, J. P. B. y Corder, S. P. (eds.) (1974): The Edinburgh Course in Applied Linguistics: Techniques in Applied Linguistics (vol. 3). Oxford: Oxford University Press.

Bachman, L. F. y Palmer, A. S. (1996). Language Testing in Practice. Oxford:

Bachman, L: F. (1990). Fundamental Considerations in Language Testing. Oxford: Oxford University Press.

Bernstein, B. (ed.) (1971). Class, Codes and Control. Volume 1. London: Routledge and Kegan Paul.

Bueno, A. (1996). "Writing”, en McLaren, N. y D. Madrid (eds) (1996): A Handbook for TEFL. Alcoy: Marfil, pp. 285-312.

Bueno, A. (2005). "Advanced Composition Techniques for Spanish Students of English Philology", GRETA, vol. 13, 1 \& 2, pp. 35-44.

Byrne, D. (1979). Teaching Writing Skills. London. Longman.

Camps, A., Guasch, O., Milian, M. y Bas, E. (2000). "Metalinguistic Activity: the link between writing and learning to write", en G. Rijlaarsdam \& E. Espéret (Series Eds.) \& A. Camps \& M. Milian (Vol Eds.), Studies in Writing: Vol. 6. Metalinguistic.

Celce-Murcia, M. (ed.) (2001). Teaching English as a second or foreign language. Boston: Heinle $\&$ Heinle.

Charles, M. (1990). "Responding to problems in written English using a student self-monitoring technique". ELT Journal 44/4: 286-293.

Chenoweth, N.A. (1987). "The need to teach re-writing". ELT Journal 41/1: 25-30.

Crewe, W. J. (1990). "The illogic of logical connectives". ELT Journal 44/4: 316-325.

Cummins, J. (1979). "Cognitive/academic language proficiency, linguistic interdependence, the optimal age question and some other matters". Working papers on Bilingualism 19, 197205.

Davies, A. (1990). Principles of Language Testing. Applied Language Studies. David Crystal and Keith Johnson (eds).

Davies, A. y Widdowson, H. G. (1974). Reading and Writing. Allen and Corder (eds) (1974)

Decreto 148/2002 de 14 de mayo, enseñanzas correspondientes a la Educación Secundaria Obligatoria en Andalucía (BOJA 27 de junio)

Dixon, D. (1986). "Teaching composition to large classes". English Teaching Forum 24/3: 2-5 and 10.

Ferris, D. (2004). "The "grammar correction" debate in L2 writing: where are we, and where do we go from here? (and what do we do in the meantime...?)". Journal of Second Language Writing, 13: 49-62. 
Flower, L. (1979). "Writer-based prose: A cognitive basis for problems in writing”. College English 41: 19-37.

Genesse, F. y Upshur, J. A. (1996). Classroom-based Evaluation in Second Language Education. Cambridge, Reino Unido: Press Syndicate of theUniversity of Cambridge.

Goldstein, L.M. (2004). "Questions and answers about teacher written commentary and student revision: teachers and students working together". Journal of Second Language Writing, 13: 63-80.

Grabe, W. and Kaplan, R. (1996).Theory and Practice of Writing. Harlow: Longman.

Hamp-Lyons, L. (ed.) (1991). Assessing second language writing in academic contexts. Westport (Connecticut). Ablex, cop.

Hedge, T. (1988). Writing. Oxford: Oxford University Press.

Hedge, T. (2000). Teaching and Learning in the Language Classroom. Oxford: Oxford University Press.

Ike, N.J. (1990). "From brainstorming to creative essay: teaching composition writing to large classes". English Teaching Forum 28/2: 41-43.

INECSE (2002). Evaluación de las enseñanza y el aprendizaje de la lengua inglesa. Educación Secundaria Obligatoria 2001. Avance de resultados. Madrid: Ministerio de Educación, Cultura y Deporte. http//www.mec.es/inecse/pubeso-lenguainglesa-pdf.

Lewitt, P.J. (1990). "How to cook a tasty essay: the secret of real rewriting". English Teaching Forum 28/1: 2-4.

Ley Orgánica 1/1990, de 3 de octubre, de Ordenación General del Sistema Educativo (BOE de 4 de octubre).

Ley Orgánica 2/2006, de 3 de mayo, de Educación (BOE de 4 de mayo).

Madrid, D y McLaren, N. (1995). Didactic Procedures for TEFL. Valladolid: La Calesa.

Manchón, R.M. (1999). "La investigación sobre la escritura como proceso. Algunas implicaciones para la enseñanza de la composición en una lengua extranjera", in Salaberri, S. (ed): Lingüística aplicada a la enseñanza de lenguas extranjeras. Almería: Servicio de Publicaciones de la Universidad, pp. 439-478.

Manchón, R.M. (2001). "Trends in the conceptualization of second language composing strategies: A critical analysis", in Manchón, R.M. (ed.): Writing in the L2 Classroom: Issues in Research and Pedagogy. International Journal of English Studies, 1, 1: 47-70.

Manchón, R., Murphy, L., Roca, J. y Aguado, P. (2005). "Learning and teaching writing in the EFL classroom", en McLaren, N., Madrid, D. y Bueno, A. (eds).: TELF in Secondary Education. Granada: Universidad de Granada.

Marco Común Europeo para las Lenguas: Aprendizaje, Enseñanza, Evaluación (2001). Consejo de Europa, Departamento de Política Lingüística. Estrasburgo.

Márquez, E.J. (1981). "Teaching grammar and paragraph structure simultaneously”. English Teaching Forum 19/3: 14-17.

McLaren, N., Madrid, D. y Bueno, A. (eds) (2005). TEFL in Secondary Education. Granada: Universidad de Granada.

Pincas, A. (1982/1983). Teaching English Writing. London: Macmillan.

Raimes, A. (1983). Techniques in Teaching Writing. New York: O.U.P.

Sampson, G.P. (1981). "A functional approach to teaching writing”. English Teaching Forum 19/ 3:10-13.

Scrivener, J. (1994). Learning teaching: a guidebook for English language teachers. Oxford: Heinemann, 1994.

Ur, P. (1996). A course in language teaching, Cambridge University Press, Cambridge.

VV.AA. (1997). Resultados de los alumnos. INCE, en www.ince.mec.es/hf/index.htm, en 
www.ince.mec.es/hf/intro.htm, en www.ince.mec.es/a.htm, en www.ince.mec.es/hf/b1.htm, en www.ince.mec.es/hf/b2.htm, en www.ince.mec.es/anx1.htm, en www.ince.mec.es/anx2.htm, y en www.ince.mec.es/anx3.htm.

White, R. V. (1980/1983). Teaching Written English. PTT. London: George Allen \& Unwin. Widdowson, H. G. (1990). Aspects of Language Teaching. Oxford: Oxford University Press. Willis, J. (1996). A Framework for Task-based Learning. London. Longman. 


\section{ANEXO I \\ Writing test}

1. Write a brief composition about a problem on a journey. First think about these questions: where did you go? when? who with? what happened? (extension: 100 words).

2. Read the following text and then write about what you wear.

My favourite clothes We asked actress, Catherine Churchill, about her favourite clothes.... "When I'm working, I never wear my own clothes, of course. I like wearing different costumes- they really help me to understand the part I'm playing. At the moment I'm filming a historical drama for TV, so I'm wearing long dresses with tight waists - very uncomfortable! When I go out with friends to a party or a restaurant, I like wearing simple elegant clothes - maybe a short black dress or trousers with a cashmere top. When I go out for a very special occasion, like a film premiere, I always wear an evening dress. When I'm relaxing at home, I wear comfortable, old clothes - a tracksuit and trainers or my blue jeans and a sweatshirt. I don't wear any make-up at all. I look very different! The great thing is - when I go out for a walk or to the shops, people usually don't recognise me!»

Now write about what you wear. Use the text above as a model (extension: 100 words).

3. Write a description of your village, town, city. (Try to use phrases expressing opinion for example: In my opinion, I think that...) (extension: 100 words)

4. Correct the grammatical errors.

- I'll switch on the lights when I will arrive

- They started to run as soon as they see me.

- The police caught a long-hair man

- Mary is a girl of blue eyes.

- If it will rain, we won't go out. 
5. Underline the synonyms or write a synonym (words of similar meaning) of the words on the left in italics.

\begin{tabular}{|c|c|c|c|c|}
\hline 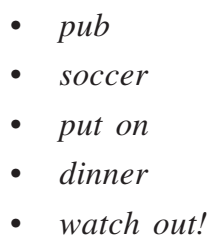 & $\begin{array}{l}\text { pet } \\
\text { sock } \\
\text { switch on } \\
\text { slim } \\
\text { Get out! }\end{array}$ & $\begin{array}{l}\text { bar } \\
\text { shoe } \\
\text { wind on } \\
\text { runner } \\
\text { Be careful! }\end{array}$ & $\begin{array}{l}\text { pool } \\
\text { football } \\
\text { come on } \\
\text { doll } \\
\text { Come out! }\end{array}$ & $\begin{array}{l}\text { puff } \\
\text { tennis } \\
\text { get on } \\
\text { supper } \\
\text { He's out! }\end{array}$ \\
\hline
\end{tabular}

- Wealthy people

- This is hard work

- She said good-by to him forever

6. Complete these sentences with:

$\begin{array}{llllll}\text { OE } & \text { UE } & \text { OO } & \text { U } & \text { OA } & \text { O }\end{array}$

- $\mathrm{A} \mathrm{c}$ ch and a 1 rry on the $\mathrm{r}$ d.

- The sky is bl and the $\mathrm{s}$ $\mathrm{n}$ is yellow.

- $\mathrm{P}$ $\mathrm{t}$ on your sh $\mathrm{s}, \mathrm{cr}$ ss the $\mathrm{w}$ d and go to sch 1 .

- Help, a b 11 !

Now write these sentences correctly:

This is the wether round the glove for todai: it is estremeli cold and snoui in alaska, nortern canada and northern rusia. There's low presure on Central América and sauthwest of india.

7. These ideas are useful to learn English.

* have a regular study routine, * make a note of your common errors, * collect useful phrases, for example, «in my opinion», * learn one irregular verb every day, * study for short periods frequently, ${ }^{*}$ never study in a room with television, * make a note of how much time you study, * learn six new words every day.

- Which ones do you use regularly?

- Which ones are you going to use in the future? 
8. Read the text and then describe a celebration.

On $5^{\text {th }}$ November people all over Britain celebrate Guy Fawkes. Before $5^{\text {th }}$ November, people build bonfires, and make models of a man with old clothes and newspaper. The man is called a «guy». Then on the evening of $5^{\text {th }}$ November we put the guy on the bonfire and light it. We also light lots of fireworks. It's great fun, but why do we do it? On $5^{\text {th }}$ November 1605 , a man called Guy Fawkes tried to kill King James I. The King was Protestant and Guy Fawkes was Catholic. He put a bomb under the Houses of Parliament in London, but soldiers discovered him and he was executed. That's why we put a model of a man on the fire - it represents Guy Fawkes.

Now describe any celebration or cultural event that has some similarities with Guy Fawkes.

9. Answer the questions.

- Do you think learning languages is important? Why?

- Do you think that English is important nowadays? Why

- Mention activities where English is used

10. Imagine you are organizing a medical congress in Brussels and you have to choose two official languages. Which ones would you choose? Why?

\section{Project Work (55 minutes)}

\section{Project: The Decade I was born}

What happened during the decade you were born? Talk about at least one important event in the world or in your country. What were the most popular music groups? Mention the people who were important in those days. Were there any important inventions? You can get information from the school library (for example, an encyclopaedia) or the Internet. 